\title{
An Estimable Dynamic Model of Entry, Exit and Growth in Oligopoly Retail Markets
}

\author{
Victor Aguirregabiria (corresponding author) \\ Department of Economics \\ University of Toronto \\ 100 Saint George Street \\ Toronto, Ontario, M5S 3G3 \\ Phone: 416-978-4358 \\ Fax: 416-978-6713 \\ victor.aguirregabiria@utoronto.ca \\ Pedro Mira \\ Centro de Estudios Monetarios y Financieros (CEMFI) \\ Casado del Alisal, 5 \\ 28014 Madrid SPAIN \\ Phone: 3491 429-0551 \\ Fax: 3491 429-1056 \\ mira@cemfi.es \\ Hernan Roman \\ Department of Economics \\ Boston University \\ 270 Bay State Road \\ Boston, MA 02205 \\ hroman@bu.edu
}

\section{AEA SESSION TITLE: EMPIRICAL INDUSTRIAL ORGANIZATION SESSION CHAIR: PATRICK BAJARI}




\section{An Estimable Dynamic Model \\ of Entry, Exit and Growth in Oligopoly Retail Markets By VICTOR AGUIRREGABIRIA, PEDRO MIRA, AND HERNAN ROMAN*}

Retail industries are an important part of today's economies. They employ a large fraction of the labor force, play a key role in the adoption and diffusion of new information technologies, and are closely related to the development and configuration of urban life. This paper presents an estimable dynamic structural model of an oligopoly retail industry. The model can be estimated using panel data of local retail markets with information on new entries, exits and the size and growth of incumbent firms. In our model, retail firms are vertically and horizontally differentiated, compete in prices, make investments to improve the quality of their businesses, and decide to exit or to continue in the market. The model extends in two important ways the entry-exit model estimated in Aguirregabiria and Mira (2007, AM hereafter). First, it includes firm size and growth as endogenous variables. And second, the empirical model has two sources of permanent unobserved heterogeneity: local-market heterogeneity and firm heterogeneity. This allows the researcher to control for potentially important sources of bias when using firm panel data with many local markets and several time periods. Not accounting for market unobserved heterogeneity can lead to biases in the estimation of those structural parameters that represent strategic interactions between firms' decisions. Market heterogeneity induces a positive correlation between firms' decisions that can be spuriously confounded with positive strategic interactions. It is also well-known in panel data econometrics that ignoring unobserved heterogeneity across firms induces biases in the parameters that generate structural state dependence, e.g., investment costs. We extend the Nested Pseudo Likelihood estimation method in AM to deal with both forms of permanent unobserved heterogeneity. This note contributes to the emerging literature on the estimation of empirical games of industry dynamics (see also Patrick Bajari, Lanier Benkard and Jonathan Levin, 2007, Martin Pesendorfer and Philipp Schmidt-Dengler, 2002, and Ariel Pakes, Michael Ostrovsky, and Steven Berry, 2007). 
In a related paper (Aguirregabiria, Mira and Roman 2006, AMR herafter) we use this model to study the sources of cross-industry heterogeneity in the dynamics of market structure. We use annual panel data from a census of Chilean firms collected by the Chilean Servicio de Impuestos Internos (Internal Revenue Service) for the period 1994-2000. For every establishment paying sales taxes, this data set reports its industry, at the five-digit level, its annual revenue, and the district where the establishment is located. Competition in retail industries occurs at the local level and we consider districts as local markets. We find large cross-industry heterogeneity along several dimensions of market structure and industry dynamics, e.g., entry and exit rates, Herfindhal index, the relationship between firm size and market size, or the relationship between firm growth and firm size. We estimate the model separately for each retail industry and use these estimates to evaluate the role that product differentiation, economies of scale, exogenous entry costs, and endogenous sunk costs play in explaining the observed cross-industry heterogeneity.

\section{The Model}

In order to better understand the role of some assumptions and features of the model, it will be helpful to describe the type of data that we will use to estimate this model. We have panel data of $M$ isolated retail markets (e.g., small towns) over $T$ years. We look at a particular retail industry, e.g., hotels, car dealers, or supermarkets. For this industry and for each of the $M$ markets, we have a panel of active firms. We observe firms' annual revenue and entry and exit decisions during the sample period. The equilibrium of our model provides predictions for the evolution of these variables in a market.

Consider a local market for a retail product. The market is populated by consumers and firms. We index firms by $i$. Time is discrete and indexed by $t$. At period $t$ there are $S_{t}$ consumers, $N_{t}^{\text {in }}$ incumbent firms operating in the market, and $N_{t}^{\text {out }}$ firms which are not active but are potential entrants, i.e., they may choose to operate in the market. We refer to $S_{t}$ as the market size, and we assume that it evolves exogenously according to a Markov process with transition probability function $f_{S}\left(S_{t+1} \mid S_{t}\right)$. The number of potential 
entrants is proportional to market size: $N_{t}^{\text {out }}=\operatorname{int}\left(\delta S_{t}\right)$, where $\delta$ is a parameter. The number of incumbent firms is endogenously determined in the equilibrium of the model. Each incumbent firm sells a differentiated product. Firms compete in prices but also in the quality of their products.

We consider a logit model of demand for differentiated products and assume that every period firms compete in prices a la Bertrand, as in Anderson, De Palma and Thisse (1992, pp. 264-266). The equilibrium of this static model of demand and price competition results in an indirect variable profit function that depends on a firm's own product quality, the competitors' qualities and market size. Let $w_{i t}$ be the quality of firm $i$ at period $t$, and let $\Omega$ be the set of possible product qualities, which is a discrete and finite set. Define the vector $n_{t}=\left\{n_{t}(w): w \in \Omega\right\}$ where $n_{t}(w)$ is the number of incumbent firms at period $t$ with quality $w$. The equilibrium variable profit of firm $i$ is $\theta_{v} S_{t} m\left(w_{i t}, n_{t}\right) s\left(w_{i t}, n_{t}\right)$ where $m\left(w_{i t}, n_{t}\right)$ and $s\left(w_{i t}, n_{t}\right)$ are the retailer's equilibrium price-cost margin and market share, respectively, and $\theta_{v}$ is a parameter that represents the degree of horizontal product differentiation.

There are two forces that affect the evolution of a retailer's stock of quality. A firm can improve its quality by making additional investments. If the firm does not invest in quality improvement, its quality depreciates as the result of increases in the value of the outside alternative. An incumbent firm's investment decision is a binary choice: $i_{i t} \in\{0,1\}$, where $i_{i t}=1$ means positive investment. The transition probability of quality for an incumbent firm is $f_{w 1}\left(w_{i, t+1} \mid w_{i t}, i_{i t}\right)$.

Incumbent firms are also heterogeneous in their investment costs. This source of heterogeneity is important in some retail industries where the cost of financing new investments varies significantly across firms. Let $\gamma_{i}$ be the firm-specific and time-invariant component of firm $i$ 's investment cost. Let $\Gamma$ be the set of possible investment costs, which is assumed discrete and finite. Define the vector $n_{t}^{*}=\left\{n_{t}^{*}(w, \gamma):(w, \gamma) \in \Omega \times \Gamma\right\}$ where $n_{t}^{*}(w, \gamma)$ is the number of incumbent firms at period $t$ with quality $w$ and investment cost $\gamma$. We refer to $n_{t}^{*}$ as the latent market structure because it depends on the distribution of the latent (i.e., 
unobserved for the researcher) investment costs.

Every period $t$, incumbent firms decide their respective investments in quality improvements and whether to remain in the market or exit at the next period. Potential entrants decide whether to enter or not in the market. Firms make these decisions to maximize expected discounted intertemporal profits $E_{t}\left(\sum_{j=t}^{\infty} \beta^{j} \Pi_{i, t+j}\right)$ where $\beta \in(0,1)$ is the discount factor and $\Pi_{i t}$ is the firm's current profit. A potential entrant that decides to stay out of the market gets zero profits. If he decides to enter, he has to pay at period $t$ an entry cost $\theta_{E C}+\varepsilon_{E, i t}$, where $\theta_{E C}$ is the component of entry costs that is common to all the firms in the market, and $\varepsilon_{E, i t}$ is a firm-specific component which is private information of the firm, has zero mean, and is i.i.d. over firms and over time. The new entrant is not active until next period. Furthermore, the firm-specific investment cost and the initial quality of a new entrant are uncertain when the firm makes its entry decision, and they are not realized until the next period. Idiosyncratic investment costs are drawn from the discrete density function $f_{\gamma}$ with support $\Gamma$. The initial quality is a random draw from the probability function $f_{w 0}$.

Current profits of an incumbent firm that stays in the market are $\theta_{v} S_{t} R\left(w_{i t}, n_{t}\right)-F C_{i t}-$ $I C_{i t}$. The first term is the variable profit. The second term is the fixed operating cost: $F C_{i t}=$ $\theta_{F C}+\varepsilon_{F C, i t}$, where $\theta_{F C}$ is a parameter and $\varepsilon_{F C, i t}$ is a private information shock. The last term, $I C_{i t}$, represents investment costs for quality improvement: $I C_{i t}=\left(\theta_{I C}+\sigma_{\gamma} \gamma_{i}+\varepsilon_{I, i t}\right) i_{i t}$, where $\theta_{I C}$ and $\sigma_{\gamma}>0$ are parameters, $\gamma_{i}$ is the firm-specific component of the investment cost, which has zero mean and unit variance, and $\varepsilon_{I, i t}$ is private information of firm $i$, has zero mean, and is i.i.d. over time and over firms. The profit of an incumbent firm that decides to exit from the market at the end of period $t$ is $\theta_{v} S_{t} R\left(w_{i t}, n_{t}\right)-F C_{i t}+\varepsilon_{X, i t}$. The exiting firm is operative during period $t$, it obtains its variable operating profits and it has to pay fixed costs. It also receives an exit value $\varepsilon_{X, i t}$ that is private information and has the same statistical properties as the other $\varepsilon^{\prime} s$. We assume that the pool of potential entrants is renewed every period. Therefore, exiting firms do not become potential entrants the following period and their continuation value is the value of the best outside alternative, which we 
normalize to zero. Likewise, the continuation value of potential entrants who choose to stay out of the market upon drawing a large entry cost is also set to zero.

We assume that firms' entry, exit and investment strategies depend only on payoff relevant state variables, i.e., Markov Perfect Equilibrium (MPE). The set $\left\{\omega_{i t}, \gamma_{i}: i=1, \ldots, N_{t}^{i n}\right\}$ is common knowledge. The vector of payoff relevant state variables of firm $i$ is $\widetilde{x}_{i t}=$ $\left(w_{i t}, \gamma_{i}, n_{t}^{*}, S_{t}, \varepsilon_{E, i t}, \varepsilon_{F C, i t}, \varepsilon_{I, i t}, \varepsilon_{X, i t}\right)$. The continuation value of an entrant or a staying incumbent as of next period is $\beta E\left[V\left(\widetilde{x}_{i t+1}\right) \mid \widetilde{x}_{i t}\right]$, where the value function $V$ is the solution of a Bellman equation. Following $A M$, we can show that a MPE can be represented in terms of players' choice probabilities conditional on common knowledge state variables. In this model, there are three free choice probability functions: the probability of entry for a potential entrant, $P_{E}\left(n_{t}^{*}, S_{t}\right)$; and, for an incumbent firm, the probability of exiting the market, $P_{X}\left(w_{i t}, \gamma_{i}, n_{t}^{*}, S_{t}\right)$, and the probability of staying and investing in quality, $P_{I}\left(w_{i t}, \gamma_{i}, n_{t}^{*}, S_{t}\right)$. Let $\mathbf{P}=\left(P_{E}, P_{X}, P_{I}\right)$ denote the vector of choice probability functions. These functions describe a firm's behavior as well as its beliefs about the behavior of its opponents. Given these beliefs, one can interpret each firm's problem as a game against nature with a unique optimal decision rule in probability space, which is the firm's best response. The equilibrium probability function is a fixed point of this best response mapping. Suppose that the private information shock $\varepsilon_{E, i t}$ has a logistic distribution with dispersion parameter $\sigma_{E}$, and that the shocks $\left\{\varepsilon_{F C, i t}, \varepsilon_{I, i t}, \varepsilon_{X, i t}\right\}$ have a type I extreme value distribution with dispersion parameter $\sigma_{X}$. Let $\tilde{\boldsymbol{\theta}}_{\pi}$ be the vector of structural parameters $\left(\theta_{v}, \theta_{F C}, \theta_{E C}, \theta_{I C}, \sigma_{\gamma}\right)^{\prime}$. Then, the best response of a firm with beliefs $\mathbf{P}$ is given by the following probability functions:

$$
\begin{aligned}
\Psi_{E}\left(\mathbf{n}_{t}^{*}, S_{t} ; \mathbf{P}\right) & =\exp \left\{\mathbf{z}_{t}^{E}\left(\tilde{\boldsymbol{\theta}}_{\pi} / \sigma_{E}\right)+\left(\sigma_{X} / \sigma_{E}\right) \lambda_{t}^{E}\right\} / \Sigma_{t}^{E} \\
\Psi_{I}\left(w_{i t}, \gamma_{i}, \mathbf{n}_{t}^{*}, S_{t} ; \mathbf{P}\right) & =\exp \left\{\mathbf{z}_{i t}^{I}\left(\tilde{\boldsymbol{\theta}}_{\pi} / \sigma_{X}\right)+\lambda_{i t}^{I}\right\} / \Sigma_{i t}^{X} \\
\Psi_{X}\left(w_{i t}, \gamma_{i}, \mathbf{n}_{t}^{*}, S_{t} ; \mathbf{P}\right) & =\exp \left\{\mathbf{z}_{i t}^{X}\left(\tilde{\boldsymbol{\theta}}_{\pi} / \sigma_{X}\right)\right\} / \Sigma_{i t}^{X}
\end{aligned}
$$

where $\Sigma_{t}^{E} \equiv 1+\exp \left\{\mathbf{z}_{t}^{E}\left(\tilde{\boldsymbol{\theta}}_{\pi} / \sigma_{E}\right)+\left(\sigma_{X} / \sigma_{E}\right) \lambda_{t}^{E}\right\}$ and $\Sigma_{i t}^{X} \equiv \exp \left\{\mathbf{z}_{i t}^{I}\left(\tilde{\boldsymbol{\theta}}_{\pi} / \sigma_{X}\right)+\lambda_{i t}^{I}\right\}+\exp \left\{\mathbf{z}_{i t}^{N I}\left(\tilde{\boldsymbol{\theta}}_{\pi} / \sigma_{X}\right)+\right.$ $\left.\lambda_{i t}^{N I}\right\}+\exp \left\{\mathbf{z}_{i t}^{X}\left(\tilde{\boldsymbol{\theta}}_{\pi} / \sigma_{X}\right)\right\}$. The vectors $\mathbf{z}_{t}^{E}, \mathbf{z}_{i t}^{I}, \mathbf{z}_{i t}^{N I}$ and $\mathbf{z}_{i t}^{X}$ and the scalars $\lambda_{t}^{E}, \lambda_{i t}^{I}, \lambda_{i t}^{N I}$ and $\lambda_{i t}^{X}$ 
are functions of the state $\left(\mathbf{n}_{t}^{*}, S_{t}\right)$ and collect the infinite sum of expected payoffs along all possible future histories originating from that state. These expected payoffs are obtained using beliefs $\mathbf{P}$ about current and future behavior and the 'primitive' probabilities $\left\{f_{w 0}, f_{w 1}, f_{\gamma}\right\}$, and they are discounted by $\beta$. Because $z^{\prime} s$ and $\lambda^{\prime} s$ depend on choice probabilities $\mathbf{P}$, the expressions in (1) describe a fixed-point mapping and the equilibrium probability functions $\left\{P_{E}, P_{X}, P_{I}\right\}$ are a fixed point of this mapping. Further details including expressions for $z^{\prime} s$ and $\lambda^{\prime} s$ can be found in AM and AMR. The model implies that market structure $n_{t}^{*}$ follows a first order Markov process.

\section{Estimation Method}

Consider that we have a sample of $M$ isolated retail markets, where $M$ is large. Our asymptotic estimation results apply when the number of markets $M$ goes to infinity. For each market $m$ we observe all the firms which are active in the market between periods 1 and $T$. For each firm $i$ we observe $\left\{e_{i m}, x_{i m}, R_{i m t}: t=e_{i m}, \ldots, x_{i m}\right\}$, where $e_{i m}$ and $x_{i m}$ are the entry and the exit periods of firm $i$, respectively, and $R_{i m t}$ is the revenue of this firm at period $t$. For the incumbents at the initial period $t=1$ we do not know their actual entry period. We represent these firms with an entry period equal to one, i.e., $e_{i m}=1$. For the firms who are still active at the end of period $T$, we do not know their actual exit period. We represent these firms with an exit period equal to $T+1$, i.e., $x_{i m}=T+1$. We also observe a measure of market size, $S_{m t}$. In AMR, we show that, under the assumption that all the operating costs are fixed, the logit model of demand and price competition can be used to obtain firms' qualities $\left\{w_{i t}\right\}$ from firms' revenue data. Therefore, we treat qualities as observable variables. We assume that: (1) a firm cannot increase his quality if he does not invest, i.e., $f_{w 1}\left(w_{i, t+1} \mid w_{i t}, i_{i t}=0\right)=0$ for $w_{i, t+1} \geq w_{i t}$; and (2) if a firm invests, then his quality does not depreciate, i.e., $f_{w 1}\left(w_{i, t+1} \mid w_{i t}, i_{i t}=1\right)=0$ for $w_{i, t+1}<w_{i t}$. Under these assumptions, a firm's quality grows if and only if the firm invests in quality improvement: i.e., $i_{i t}=I\left\{w_{i, t+1} \geq w_{i t}\right\}$. Therefore, investment is also observable for every period $t \leq T-1$. The number of potential entrants is estimated as $\hat{N}_{m t}^{o u t}=\operatorname{int}\left(\hat{\delta} S_{m t}\right)$, where $\hat{\delta}=\max _{m, t}\left\{\bar{e}_{m t} / S_{m t}\right\}$ 
is a consistent estimator of $\delta$, and $\bar{e}_{m t}$ is the number of entrants at period $t$.

The structural parameters in the profit function are identified only up to scale. We use $\boldsymbol{\theta}_{\pi}$ to denote the vector of identified structural parameters: $\boldsymbol{\theta}_{\boldsymbol{\pi}} \equiv\left(\tilde{\boldsymbol{\theta}}_{\pi}^{\prime} / \sigma_{X}, \sigma_{E} / \sigma_{X}\right)$. Let $\boldsymbol{\theta}_{f}$ be the vector of structural parameters that characterizes the probability functions $f_{S}, f_{w 0}$ and $f_{w 1}$. Note that $\gamma_{i}$ is a standardized random variable, i.e., the mean and the variance of the heterogenous investment costs are $\theta_{I C}$ and $\sigma_{\gamma}^{2}$, respectively. We assume that the distribution $f_{\gamma}$ of the standardized heterogeneity $\gamma_{i}$ is known to the econometrician, e.g., it is a discretized version of the density of a standard normal. That is, we consider distributions of unobserved firm heterogeneity with only two parameters, mean and variance, such that the distribution of the standardized heterogeneity is known. This assumption simplifies very importantly the computation of the maximum pseudo likelihood estimator in our procedure because it implies global concavity of the pseudo likelihood. Given this assumption, it is possible to incorporate firm heterogeneity in any of the other parameters of the model (e.g., $\theta_{v}, \theta_{F C}$ or $\left.\theta_{E C}\right)$ keeping the global concavity of the pseudo likelihood function.

The likelihood function of this model and data has the form $\prod_{m=1}^{M} L_{m}\left(\boldsymbol{\theta}_{\pi}, \boldsymbol{\theta}_{f}\right)$, where $L_{m}\left(\boldsymbol{\theta}_{\pi}, \boldsymbol{\theta}_{f}\right)$ is the contribution of market $m$ to the likelihood function. We derive here the expression of the likelihood $L_{m}$. For notational simplicity, we omit the market subindex $m$. Also, for the sake of space, we ignore here unobserved market heterogeneity and concentrate in firm heterogeneity (see $A M R$ for the description of the NPL with both types of unobserved heterogeneity). Equilibrium probabilities depend on the firm's own type and on the types of all the incumbent firms. Therefore, in order to obtain the likelihood function, we have to integrate over the distribution of firms' types. Let $\tilde{\gamma} \equiv\left\{\gamma_{i}: i=1,2, \ldots, N\right\}$ be the vector with the (unobserved) type of each firm in the sample. Then, $L\left(\boldsymbol{\theta}_{\pi}, \boldsymbol{\theta}_{f}\right)=\sum_{\tilde{\boldsymbol{\gamma}} \in \boldsymbol{\Gamma}^{N}} \operatorname{Pr}(\tilde{\boldsymbol{\gamma}})$ $\operatorname{Pr}($ Data $\mid \tilde{\gamma})$, and $\operatorname{Pr}($ Data $\mid \tilde{\gamma})=L_{1}\left(\boldsymbol{\theta}_{f}\right) L_{2}\left(\boldsymbol{\theta}_{\pi}, \boldsymbol{\theta}_{f} \mid \tilde{\gamma}\right)$. The function $L_{1}\left(\boldsymbol{\theta}_{f}\right)$ is the likelihood of the history of market sizes and firms' qualities (conditional on investment decisions):

$$
L_{1}\left(\boldsymbol{\theta}_{f}\right)=\left[\prod_{i: e_{i} \neq 1} f_{w 0}\left(w_{i e_{i}}\right) \prod_{i=1}^{N} \prod_{t=e_{i}+1}^{x_{i}} f_{w 1}\left(w_{i t} \mid w_{i, t-1}, i_{i, t-1}\right)\right]\left[\prod_{t=2}^{T} f_{S}\left(S_{t} \mid S_{t-1}\right)\right]
$$

This likelihood depends only on the sub-vector $\boldsymbol{\theta}_{f}$ of the structural parameters, and it does 
not depend on the unobserved types $\tilde{\boldsymbol{\gamma}}$. The function $L_{2}\left(\boldsymbol{\theta}_{\pi}, \boldsymbol{\theta}_{f} \mid \tilde{\gamma}\right)$ is the likelihood of the initial conditions $\left(\mathbf{n}_{1}, S_{1}\right)$ and of the history of firms' entry, exit and investment decisions conditional on the vector of firm types $\tilde{\gamma}$ :

$$
\begin{aligned}
& L_{2}\left(\boldsymbol{\theta}_{\pi}, \boldsymbol{\theta}_{f} \mid \tilde{\gamma}\right)=\operatorname{Pr}\left(\mathbf{n}_{1}, S_{1} \mid \tilde{\gamma}_{1}\right) \quad\left[\prod_{t=2}^{T} P_{E}\left(\mathbf{n}_{t}^{*}, S_{t}\right)^{\bar{e}_{t}}\left(1-P_{E}\left(\mathbf{n}_{t}^{*}, S_{t}\right)\right)^{N_{t}^{\text {out }}-\bar{e}_{t}}\right] \\
& {\left[\prod_{i=1}^{N} \prod_{t=e_{i}+1}^{x_{i}} P_{N I}\left(w_{i t}, \gamma_{i}, \mathbf{n}_{t}^{*}, S_{t}\right)^{1-i_{i t}} P_{I}\left(w_{i t}, \gamma_{i}, \mathbf{n}_{t}^{*}, S_{t}\right)^{i_{i t}}\left[\frac{P_{X}\left(w_{i x_{i}}, \gamma_{i}, \mathbf{n}_{x_{i}}^{*}, S_{x_{i}}\right)}{P_{N I}\left(w_{i x_{i}}, \gamma_{i}, \mathbf{n}_{x_{i}}^{*}, S_{x_{i}}\right)}\right]^{I\left\{x_{i} \leq T\right\}}\right]}
\end{aligned}
$$

where $P_{N I}$ is the probability of staying in the market without new investments, and $\tilde{\gamma}_{1}$ is the vector of types of the incumbent firms at the beginning of $t=1$. The probability $\operatorname{Pr}\left(\mathbf{n}_{1}, S_{1} \mid \tilde{\gamma}_{1}\right)$ represents the so-called initial conditions problem. This probability can be obtained easily from the steady-state equilibrium distribution of the state variables $\left(\mathbf{n}_{t}^{*}, S_{t}\right)$, i.e., $p^{*}\left(\mathbf{n}_{t}^{*}, S_{t}\right)$. This steady-state distribution depends on the equilibrium probabilities $\mathbf{P}$ and on the primitives $\left\{f_{S}, f_{w 0}, f_{w 1}\right\}$ and it can be derived by solving a system of linear equations (see $A M$ for more details). A property of $\operatorname{Pr}\left(\mathbf{n}_{1}, S_{1} \mid \tilde{\gamma}_{1}\right)$ that is key for the globall concavity of the pseudo likelihood function that we define below, is that it depends on the parameters $\boldsymbol{\theta}_{\boldsymbol{\pi}}$ only through the equilibrium probabilities $\mathbf{P}$.

Given this structure of the full likelihood, we can write the log-likelihood as the sum of two components: $\log L\left(\boldsymbol{\theta}_{\pi}, \boldsymbol{\theta}_{f}\right)=\log L_{1}\left(\boldsymbol{\theta}_{f}\right)+\log \left\{\sum_{\tilde{\gamma}} \operatorname{Pr}(\tilde{\boldsymbol{\gamma}}) L_{2}\left(\boldsymbol{\theta}_{\pi}, \boldsymbol{\theta}_{f}, \tilde{\boldsymbol{\gamma}}\right)\right\}$. Following a standard approach in the estimation of dynamic structural models, we consider partial maximum likelihood estimation. In a first step, we obtain the (partial) maximum likelihood estimator of $\boldsymbol{\theta}_{f}$ that maximizes $\log L_{1}\left(\boldsymbol{\theta}_{f}\right)$. Then, given $\hat{\boldsymbol{\theta}}_{f}$, we consider the estimation of $\boldsymbol{\theta}_{\pi}$ using the partial likelihood $\log \left\{\sum_{\tilde{\gamma}} \operatorname{Pr}(\tilde{\gamma}) L_{2}\left(\boldsymbol{\theta}_{\pi}, \hat{\boldsymbol{\theta}}_{\mathbf{f}}, \tilde{\boldsymbol{\gamma}}\right)\right\}$. The first step is a standard problem of maximum likelihood estimation and it does not require one to solve the dynamic game. We discuss here the estimation of $\boldsymbol{\theta}_{\pi}$ in the second step.

There are three main econometric and computational issues that we have to deal with to estimate this model: multiple equilibria; the computational cost associated with the repeated computation of an equilibrium of the model; and integration over all possible values of $\tilde{\gamma}$. The first two problems were the main concern in $A M$. To deal with these 
problems we propose a procedure, that we called Nested Pseudo Likelihood (NPL) that avoids the repeated solution of the game and that can be used when the model has multiple equilibria. The main idea of this procedure is relatively simple. While equilibrium probabilities are not unique functions of structural parameters, the best response probabilities $\Psi \equiv\left\{\Psi_{E}, \Psi_{I}, \Psi_{X}\right\}$, in equation (1), are always unique functions of structural parameters and firms' beliefs. We use these best response functions to construct a pseudo likelihood function $Q\left(\boldsymbol{\theta}_{\pi}, \mathbf{P}\right) \equiv \log \left\{\sum_{\tilde{\gamma}} \operatorname{Pr}(\tilde{\gamma}) L_{2}^{\Psi}\left(\boldsymbol{\theta}_{\pi}, \hat{\boldsymbol{\theta}}_{\mathbf{f}}, \mathbf{P} \mid \tilde{\gamma}\right)\right\}$, where $L_{2}^{\Psi}()$ is the likelihood in equation (3) but where we have replaced equilibrium probabilities by best response probabilities $\boldsymbol{\Psi}$, and $\mathbf{P}$ is the vector of beliefs that we use to evaluate firms' best responses. If the pseudo likelihood function is based on a consistent nonparametric estimator $\hat{\mathbf{P}}$ of the true equilibrium beliefs in the population, we can get a two-step estimator that is consistent and asymptotically normal. However, this two-step method cannot be applied to models with market or firm unobserved heterogeneity because consistent nonparametric estimates of choice probabilities are not available for these models. Instead, $A M$ propose the NPL that is a recursive extension of this two-step method. The NPL procedure starts with an arbitrary vector $\hat{\mathbf{P}}_{0}$. Given these initial probabilities, we generate a sequence of $\left\{\hat{\boldsymbol{\theta}}_{\pi, k}, \hat{\mathbf{P}}_{k}\right\}$ such that: (1) $\hat{\boldsymbol{\theta}}_{\pi, k}=\arg \max _{\boldsymbol{\theta}_{\pi}} Q\left(\boldsymbol{\theta}_{\pi}, \hat{\mathbf{P}}_{k-1}\right)$; and (2) $\hat{\mathbf{P}}_{k}=\boldsymbol{\Psi}\left(\hat{\boldsymbol{\theta}}_{\pi, k}, \hat{\mathbf{P}}_{k-1}\right)$. Upon convergence, this procedure provides a consistent estimator of the structural parameters. Step (1) is a pseudo ML estimation and it is very simple task in our model. Given $\hat{\mathbf{P}}_{k-1}$ we can construct the $z^{\prime} s$ and $\lambda^{\prime} s$ that appear in the best response probability functions. Furthermore, we use these probabilities to construct the steady-state distribution of $\left(\mathbf{n}_{t}^{*}, S_{t}\right)$ and then the probability of the initial conditions $\operatorname{Pr}\left(\mathbf{n}_{1}, S_{1} \mid \tilde{\gamma}_{1}\right)$, which are fixed during the pseudo-ML estimation. Using this approach, our pseudo likelihood function $Q\left(\boldsymbol{\theta}_{\pi}, \mathbf{P}\right)$ is globally concave in $\boldsymbol{\theta}_{\pi}$ for any value of $\mathbf{P}$, which facilitates very much the computation of the procedure, and guarantees the consistency of the NPL estimator. Step (2) is a policy iteration, and it consists in the evaluation of the expressions in the right hand sides of equation (1) using the $z^{\prime} s$ and $\lambda^{\prime} s$ from previous iteration $k-1$ but the new value of the structural parameters $\hat{\boldsymbol{\theta}}_{\pi, k}$. 
A new computational issue that appears when dealing with unobserved firm heterogeneity is that we have to integrate over $\tilde{\gamma}$. The dimension of the set $\Gamma^{N}$ of possible values of $\tilde{\gamma}$ increases exponentially with the number of firms that we observe in a market, $N$. For most oligopoly markets, $N$ is small and integration over all $\tilde{\gamma}^{\prime} s$ may be computationally inexpensive. For instance, with $N=10$ and two types, the number of possible values of $\tilde{\gamma}$ is just $2^{10}=1,024$. However, with two types and $N \geq 20$ we have more than one million of possible values for $\tilde{\gamma}$. In these cases, simulation techniques can be used to integrate over the distribution of types. Simulation can be used also to approximate the solution to the system of linear equations that defines the $\mathbf{z}^{\prime} s$ and $\lambda^{\prime} s$ in firms' best response probabilities, as in Bajari, Benkard, and Levin (2007). The combination of these simulation techniques, that reduce further the cost of estimating these models, with the NPL method, that permits one to deal with unobserved heterogeneity, is an interesting topic for further research.

\section{REFERENCES}

Aguirregabiria, Victor and Mira, Pedro. "Sequential Estimation of Dynamic Discrete Games," Econometrica, 75, 1-53. January, 2007.

Aguirregabiria, Victor; Mira, Pedro; and Roman, Hernan. "Entry, Survival and Growth in Oligopoly Retail Markets: Explaining Cross-Industry Heterogeneity." Unpublished manuscript, University of Toronto. September 2006.

Anderson, Simon; De Palma, Andre; and Thisse, Jean-Francois. "Discrete Choice Theory of Product Differentiation." The MIT Press. Cambridge, Massachusetts. 1992.

Bajari, Patrick; Benkard, Lanier; and Levin, Jonathan. "Estimating Dynamic Models of Imperfect Competition." Econometrica, forthcoming. 2007.

Pakes, Ariel; Ostrovsky, Michael; and Berry, Steven. "Simple Estimators for the Parameters of Discrete Dynamic Games (with Entry/Exit Examples)." RAND Journal of Economics, forthcoming.

Pesendorfer, Martin and Schmidt-Dengler, Philipp. "Asymptotic Least Squares Estimators for Dynamic Games." Unpublished manuscript. LSE. 2002. 